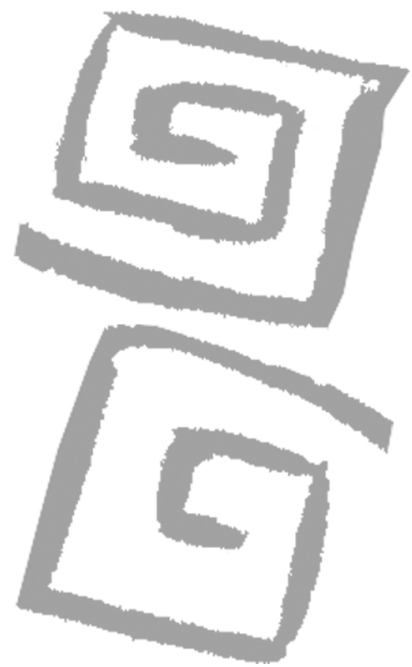

\title{
El reconocimiento de la producción subjetiva del cuidado
}

\author{
Recognizing the subjective \\ production of care
}

Franco, Túlio Batista ${ }^{1}$; Merhy, Emerson Elias ${ }^{2}$

${ }^{1}$ Doctor en Salud Colectiva. Profesor Adjunto, Universidade Federal Fluminense (UFF), Brasil. tuliofranco@gmail.com

${ }^{2}$ Doctor en Salud Colectiva. Profesor Libre Docente, Universidade Estadual de Campinas (Unicamp), Brasil. emerson.merhy@gmail.com
RESUMEN Este artículo parte del supuesto de que hay una producción subjetiva del cuidado en salud por lo cual sugiere que la investigación cualitativa en salud debe contemplar también estudios sobre esta dimensión de la realidad. La producción subjetiva del cuidado en salud se manifiesta en tres planos: el primero, las redes rizomáticas que se forman y actúan en flujos de intensidades al interior de los servicios de salud y conectan a los trabajadores en el ambiente del trabajo a través de líneas de cuidado integral; el segundo, el deseo que se forma en los procesos primarios, inconscientes, y opera como una energía de producción propulsora de los procesos de construcción de la realidad; y tercero, el "trabajo vivo en acto" que actúa como una plataforma sobre la cual se producen las redes del cuidado. Es un movimiento combinado y sinérgico entre las tres dimensiones. Esto nos revela que los trabajadores construyen su proceso de trabajo de acuerdo con un modo singular de significar el mundo e intervenir en él, poniendo un límite a las directrices normativas que intentan encuadrar las prácticas del cuidado en fórmulas rígidamente protocolares. El plano del trabajo y del cuidado en salud es el lugar de manifestación de las singularidades y por ello es diverso, múltiple, como expresión de las subjetividades en acción.

PALABRAS CLAVE Servicios de Salud; Trabajo; Evaluación de Proceso (Atención de Salud); Sistema de Salud; Brasil.

ABSTRACT This article assumes that there is a subjective production implicit in health care and suggests that qualitative investigation in health should also take into account studies on this dimension of reality. The subjective production of health care manifests itself on three levels: the first, the rizomatic networks which are formed and act in flows of intensities within health services and connect the workers in their working environment through lines of integral care; the second, the desire which is formed in primary, unconscious processes and operates as a productive energy to propel the processes that construct reality; and the third, the "live work in action" which serves as a platform upon which the networks of health care are produced. There is a combined and synergic movement among the three dimensions. This tells us that workers construct their work processes according to a unique way of understanding the world and taking part in it, putting a limit on the normative frameworks that attempt to structure the practice of care into rigid protocols. It is within the work of caring for health that these personal singularities manifest themselves and for this reason is a diverse, varied expression of manifold subjectivities in action.

KEY WORDS Health Services; Work; Process Assessment (Health Care); Health System; Brazil. 


\section{INTRODUCCIÓN}

Este ensayo busca reunir evidencias de que hay una producción subjetiva del cuidado en salud, y a partir de esta constatación sugiere que los procesos de evaluación cualitativa de servicios de salud admitan en su plano analítico la subjetividad como una de las dimensiones del modo de producción en salud.

Se parte del supuesto de que los trabajadores de un mismo Equipo de Salud Familiar (ESF) actúan de un modo singular en la producción del cuidado, es decir, cada uno de ellos actúa de forma diferente aunque estén bajo la misma directriz normativa. Esto revela que el proceso de trabajo no sigue un patrón, pues las prácticas de cuidado están mediadas por la singularidad de cada uno. En ese escenario, las normas de los ESF, que tienen como atributo estandarizar las conductas de los trabajadores de acuerdo a reglas dictadas para el funcionamiento del programa, influyen sobre la actividad de los trabajadores dentro de límites muy restringidos, pues cuando se encuentran en situación de trabajo, en relación con el usuario, son ellos mismos los que, en acto, definen cómo se realiza ese cuidado. De ese modo, la capacidad de los niveles gestores de influir sobre la acción cotidiana de cada trabajador es reducida, y muy diferenciada.

Se percibe que el modo de producción del cuidado se revelaría de forma eficaz en el ámbito de su micropolítica, si hubiera un método capaz de verificar el dinámico y complejo modo operativo de cada trabajador en su acción cotidiana, incluso su producción subjetiva en acto, que produce el cuidado en salud $y$, al mismo tiempo, produce al propio trabajador en tanto sujeto en el mundo.

Este texto se basa, inicialmente, en la producción teórica que inscribe la subjetividad que actúa en la construcción del socius, es decir, el microcosmos en que cada uno está inserto y donde opera micropolíticamente. Al mismo tiempo se verifica que para identificar la acción micropolítica es necesaria una mirada especializada, vibrante, posible de lograr en base a ciertos instrumentos cartográficos. Estos instrumentos tienen sensores con un alto grado de sensibilidad para el estudio y comprensión de la realidad social, de la percepción de los fenómenos que la rodean y sobre todo de la producción de vida en el cotidiano basada en el protagonismo de los sujetos en acto (1-5).

La producción subjetiva del medio en que se vive y se trabaja está marcada por una constante deconstrucción y construcción de territorios existenciales, en base a ciertos criterios que son dados por el saber, pero también y fundamentalmente siguiendo la dimensión sensible de percepción de la vida y de sí mismo, en flujos de intensidades continuas entre los sujetos que actúan en la construcción de la realidad social. Esta percepción, según la cual los sujetos en la escena de trabajo actúan con flujos de conexión entre sí, está inspirada en la idea de "rizoma", usada por Deleuze y Guattari en la introducción del libro Mil mesetas (1), y expresa un movimiento de flujo horizontal y circular al mismo tiempo, que vincula lo múltiple, lo heterogéneo, dentro de la dimensión micropolítica de construcción de un mapa que está siempre abierto, permitiendo diversas entradas, y que al romperse en determinado punto, se rehace encontrando nuevos flujos que permiten su crecimiento, estableciendo nuevas conexiones en el proceso. Las mesetas, por lo tanto, aparecen como un movimiento dinámico que opera en conexión entre diversos planos de existencia y de intensidades.

El desafío de la evaluación cualitativa, según la dimensión subjetiva, es el de profundizar la comprensión de la dinámica micropolítica de cada trabajador para percibir cómo, en su singularidad, ellos producen el cuidado en el cotidiano, partiendo del supuesto de que esta producción subjetiva del cuidado en salud está presente en el ámbito del proceso de trabajo, y al mismo tiempo en la producción de sí mismos como sujetos del trabajo. Esto se produce en la medida en que interactúan con los usuarios y los problemas de salud, a través de su proceso productivo.

\section{INSTRUMENTOS CARTOGRÁFICOS APLICADOS A LA EVALUACIÓN CUALITATIVA DEL TRABAJO EN SALUD}

Preliminarmente los instrumentos cartográficos que serán discutidos aquí, están basados en tres conceptos, considerados constitutivos del 
método o que componen de manera fundamental la cartografía y le dan una identidad conceptual. Los elementos en discusión son: la tesis del rizoma como flujos de intensidades en conexión, que producen la realidad social (1), y que parece ser el concepto fundante de la idea general de la cartografía; el deseo como fuerza propulsora de la acción de los sujetos, que expresa la producción subjetiva del socius según proponen Deleuze y Guattari en El Anti Edipo (2); y por último la teoría del "trabajo vivo en acto" de Emerson Merhy $(6,7)$ como el eje de tensión que produce las líneas cartográficas. Estos tres conceptos, dentro de la cartografía que se pretende producir, son inmanentes entre sí.

\section{El rizoma: cartografía en acto en el proceso de trabajo}

La primera referencia importante para pensar en la cartografía como método de investigación, proviene de Deleuze y Guattari, en especial de la introducción del libro Mil mesetas: capitalismo y esquizofrenia, donde los autores discuten el rizoma como dispositivo de producción de la realidad social, basándose en la acción de los sujetos en conexión entre sí y con el mundo, a través de flujos de intensidades. Allí enumeran varias características de un rizoma:

$1 .^{\circ}$ y $2 .^{\circ}$ Principios de conexión y de heterogeneidad: cualquier punto del rizoma puede ser conectado con cualquier otro, y debe serlo [...] 3. ${ }^{\circ}$ Principio de multiplicidad: sólo cuando lo múltiple es tratado efectivamente como sustantivo, multiplicidad, deja de tener relación con lo Uno como sujeto o como objeto, como realidad natural o espiritual, como imagen y mundo. [...] 4. ${ }^{\circ}$ Principio de ruptura asignificante: [...] Un rizoma puede ser roto, interrumpido en cualquier parte, pero siempre recomienza según ésta o aquellas de sus líneas y según otras. [...] $5 .^{\circ}$ y $6 .^{\circ}$ Principio de cartografía y de calcomonía: un rizoma no responde a ningún modelo estructural o generativo. [...] Una de las características más importantes del rizoma quizá sea la de tener siempre múltiples entradas. (1 p.13-18)
El rizoma opera teniendo a las mesetas como plataformas de producción subjetiva del medio social, de alta intensidad, que se conectan con los planos sobre los cuales la realidad se manifiesta. Así, las mesetas toman un lugar importante en las cartografías, pues se constituyen como lugar de potencia en la producción del mundo y de la vida. Según los autores:

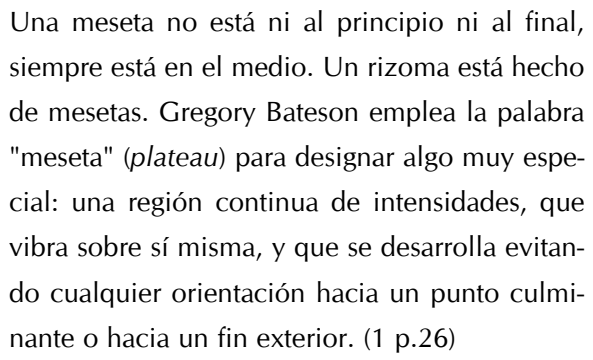

La meseta, en la microfísica del trabajo en salud, es ante todo un lugar de producción, y como tal, de encuentros de intensidades que afectan a los sujetos que están en situación de trabajo y cuidado; y el rizoma, su cartografía en acto. El carácter múltiple, heterogéneo y de muchas entradas del rizoma, le va dando porosidad y es atravesado por diversas lógicas en los agenciamientos de construcción de la realidad social. En su cartografía no caben juicios de valor sobre la actuación de los sujetos, sino que lo que se busca es comprender su funcionamiento dentro de la acción productivo-deseante que emprenden en la producción del cuidado. Dentro de esa lógica no existen el bien o el mal, lo bonito o lo feo, sino subjetividades que están capturadas por determinado territorio existencial y expresan el mundo de la vida según ese territorio. De ese modo, esos sujetos actúan según los planos de consistencia que se forman en la relación con el otro, en su alteridad inmediata y siempre en acto.

En el encuentro entre el trabajador y el usuario, ambos tienen la capacidad de afectarse el uno al otro, y esto se da por las intensidades que circulan entre las relaciones que se establecen entre los dos cuerpos, entendiendo como cuerpos a los sujetos en acción como trabajador y usuario, o trabajador y trabajador, pero también de ellos con las normas, saberes, instrumentos que configuran cuerpos en el escenario del cuidado en salud. Según Espinosa, citado por 
Deleuze (8), los afectos pueden causar alegría o tristeza, aumentando o disminuyendo respectivamente la potencia de los sujetos de actuar en el mundo de la vida. En el caso específico de la salud, las conexiones entre los diversos procesos de trabajo que se realizan entre trabajadores-trabajadores y trabajadores-usuarios pueden formar un campo energético, invisible, que funciona en flujos circulantes que envuelven al cuidado en acto y configuran "líneas de vida" o "líneas de muerte", según si el encuentro trabajador-usuario produce acogimiento, vínculo, autonomía, satisfacción, o un modo de actuar que se manifieste de forma acotada, burocrática, produciendo heteronomía, insatisfacción. Dependiendo de la situación existente habrá un aumento o reducción de la potencia de actuar.

\section{El deseo: fuerza propulsora de la acción productiva del trabajo en salud}

La segunda referencia importante para el uso de instrumentos cartográficos se refiere al concepto de deseo que se encuentra en el pensamiento fundante del esquizoanálisis.

En el libro El Anti Edipo: capitalismo y esquizofrenia, Deleuze y Guattari (2) entablan una lucha conceptual, al estilo de las grandes epopeyas, para afirmar la idea según la cual el deseo que se forma en el inconsciente es energía productiva y por lo tanto, impulsora de la construcción de la realidad social por el sujeto. Según Deleuze y Guattari:

\footnotetext{
...la primera evidencia es que el deseo no tiene por objeto a personas o cosas, sino medios enteros que recorre, vibraciones y flujos de todo tipo que desposa, introduciendo cortes, capturas, deseo siempre nómada y emigrante cuya característica primera es el "gigantismo": nadie mejor que Charles Fourier lo ha mostrado. En resumen, los medios sociales tanto como los biológicos son objeto de catexis del inconsciente que necesariamente son deseantes o libidinales, por oposición a las catexis preconscientes de necesidad e interés. (2 p.302)
}

Según los autores, el deseo, como producción, tiene la energía de la invención de la realidad social, de la creación de un nuevo devenir para el mundo de la vida: es revolucionario. El deseo es agenciamiento, es decir, está siempre en actividad, produciendo lo "real social" en toda dimensión de la vida. Esta idea se asocia a otra, según la cual la producción del mundo se da a través de subjetividades deseantes que al operar en flujos, en conexión entre muchos campos de intensidades, conforman nuevos mundos que se van constituyendo en el proceso.

La fuerza motriz de construcción de la sociedad es el deseo, que se forma a nivel inconsciente y es constitutivo de las subjetividades, y que en el plano social transforma a los sujetos en protagonistas por excelencia de los procesos de cambio. Estos mismos sujetos operan en la construcción y deconstrucción de mundos, proceso en el que se modifican los territorios existenciales. "El deseo pertenece al orden de la producción, toda producción es a la vez deseante y social" (2 p.306).

Un deseo "siempre nómade y migrante", según nos dicen los autores, que va agenciando la formación de mundos, y también su deconstrucción. Ese proceso es discutido en detaIle por Rolnik (3) cuando revela los procesos de territorialización, desterritorialización y reterritorialización -tratados aquí como territorios existenciales- y cómo ciertos acontecimientos agencian cambios en la subjetividad, ocasión en que individuos o colectivos de sujetos se desterritorializan expresando cambios estructurales en el modo de significar e interactuar con el mundo de la vida. Esto se produce por la fuerza del deseo, es decir, la fuerza motriz de producción de la sociedad, de nuevos modos de actuar en el mundo, y de producción de nuevos sujetos.

Es así como se producen las cartografías. Percibimos hasta aquí que el rizoma como flujos continuos y el deseo como fuerza productiva van componiendo una idea de formación cartográfica de los procesos de producción del cuidado. Hasta aquí, estamos buscando las referencias para un método de análisis de la producción del cuidado que revele la acción productiva de los sujetos, en tanto singularidades, y sus agenciamientos en la construcción de la realidad social del campo de la acción en el mundo del cuidado en salud. El método busca cartografiar el plano visible e invisible de los procesos de producción, 
con los sujetos en acción, impulsados por la energía deseante. Para finalizar la composición del método sugerido, traemos a esta discusión el concepto de "trabajo vivo en acto".

En su texto de 1997, "Em busca do tempo perdido: a micropolítica do trabalho vivo em saúde", Merhy recupera para la salud colectiva el concepto y la potencia del trabajo vivo, caracterizándolo como un proceso agenciado por sujetos, que trae en sí el atributo de la libertad, de la creación, de la inventiva. Naturalmente que el proceso productivo de la salud es contradictorio y el trabajo vivo puede ser capturado por la lógica instrumental de producción del cuidado: el trabajo muerto. Pero la importancia de este concepto aplicado al proceso de trabajo en salud revela la posibilidad que tienen los trabajadores de realizar su trabajo con un alto grado de libertad, ejerciendo, podemos decirlo así, un razonable autogobierno sobre su actividad productiva.

En la micropolítica del proceso de trabajo no cabe la noción de impotencia, pues si el proceso de trabajo está siempre abierto a la presencia del trabajo vivo en acto, es porque él puede ser siempre "atravesado" por diferentes lógicas que el trabajo vivo comprende. Ejemplo de ello es la creatividad permanente del trabajador en acción en la dimensión pública y colectiva, que puede ser "explotada" para inventar nuevos procesos de trabajo, y hasta para extenderlo hacia direcciones no pensadas. (7 p.44)

Según el autor, el proceso de trabajo en salud es siempre relacional, y esta relación tiene la característica del intercesor, es decir:

\footnotetext{
...lo que se produce en las relaciones entre "sujetos", en el espacio de sus intersecciones, que es un producto que existe para los "dos" en acto y no tiene existencia sin el momento de la relación, en la cual los inter se colocan como instituyentes en la búsqueda de nuevos procesos, tal cual uno en relación al otro. (7 p.37)
}

Este proceso relacional es impulsado por la libertad inmanente al "trabajo vivo en acto", y va operando relaciones en flujos de alta intensidad al interior del proceso de trabajo. Estos flujos producen una conexión entre trabajadores, usuarios, personas y cosas, que se encuentran en el plano del proceso de producción del cuidado y son partes constitutivas del mismo. Esa red que se forma en la informalidad del cotidiano de un equipo o unidad de salud, es como el rizoma, no tiene comienzo ni fin, se conecta en cualquier punto.

La libertad constitutiva del "trabajo vivo en acto", asociada a los agenciamientos del deseo inmanentes a la actividad productiva de cada trabajador, van produciendo la realidad social inscripta en el mundo del cuidado. El trabajo en salud se producirá circunscripto a un determinado territorio existencial que opera en un referencial ético-político que los trabajadores adoptan como "plano de consistencia" entre ellos y los usuarios. Este plano de consistencia se refiere a los flujos circulantes en la relación que se establece entre trabajador y usuario, y se refiere al campo invisible del cuidado en salud, los afectos que dan significado a la relación trabajadorusuario y al propio cuidado que se realiza.

\section{LA CARTOGRAFÍA: PRODUCCIÓN SUBJETIVA DE LA MICROPOLÍTICA}

\author{
De acuerdo con Kastrup (5):
}

La cartografía es un método formulado por G. Deleuze y F. Guattari (1995) que apunta a acompañar un proceso, y no a representar un objeto. En líneas generales, se trata siempre de investigar un proceso de producción. Antes de nada, la idea de desarrollar el método cartográfico para su utilización en investigaciones de campo en el estudio de la subjetividad se distancia del objetivo de definir un conjunto de reglas abstractas a ser aplicadas. No se busca establecer un camino lineal para alcanzar un fin. La cartografía es siempre un método ad hoc. (5 p.15) (a)

Es importante reafirmar que esa producción de la que habla la autora, se refiere a la producción subjetiva de la realidad social, impulsada por el deseo. Ahora bien, al producir el mundo, se está siempre en relación con el socius, lo que significa operar también y simultáneamente la producción de sí mismo. Y esa producción 
de subjetividad se da por "factores de afectivación", es decir, acontecimientos que impactan en el microcosmos y que de alguna forma Ilegan hasta el sujeto e impactan sobre su forma de significar el mundo. En este contexto hay un proceso de formación subjetiva del medio social y de sí.

Dentro del campo de la evaluación en salud, tenemos como supuesto que el trabajo de análisis extrae del mundo un conocimiento que puede retornar al mismo medio social bajo la forma de intervención sobre la realidad, y cambios que se procesan en el ambiente de interacción de los propios sujetos que están siendo analizados junto a los servicios de salud. Al mismo tiempo que modifican el medio social, los evaluadores son también afectados por su objeto, por lo tanto, se produce un proceso de subjetivación, en este caso una producción de sí. Como método de investigación, la cartografía provoca al mismo tiempo análisis e intervención, pues reconoce el proceso de producción de sí y del mundo como algo simultáneo, legítimo e inexorable.

Rolnik (3) utiliza la cartografía para analizar el Brasil de los años '80. El libro en el que publica ese trabajo tiene una primera parte en la que la autora delimita el campo teórico en el cual realiza su estudio, y en la segunda parte presenta la cartografía de la coyuntura brasileña. En el texto la autora en su singularidad define la cartografía de este modo:

Para los geógrafos, la cartografía - a diferencia del mapa, representación de un todo estático- es un dibujo que acompaña y se hace al mismo tiempo que los movimientos de transformación del paisaje.

Los paisajes psicosociales también son cartografiables. La cartografía, en ese caso, acompaña y se hace al mismo tiempo que el derrumbamiento de ciertos mundos -su pérdida de sentido- y la formación de otros: mundos que se crean para expresar afectos contemporáneos, con relación a los cuales los universos vigentes se hicieron obsoletos.

Al ser tarea del cartógrafo darle voz a los afectos que piden paso, de él se espera básicamente que esté sumergido en las intensidades de su tiempo y que, atento a los lenguajes que encuentra, devore aquellos elementos que le parezcan posibles para la composición de las cartografías que resulten necesarias. El cartógrafo es ante todo un antropófago. (3 p.23)

La autora menciona los procesos de formación y deconstrucción de territorios, entendiéndolos como "territorios existenciales", es decir, aquello que cada uno tiene dentro, y que define su forma de significar e interactuar con el mundo. Ese actuar ante la vida es singular, o sea, de cada uno, y por eso mismo es múltiple, pues tenemos siempre tantos mundos como aquellos que lo habitan. Llevándolo a la discusión de la producción del cuidado, podemos imaginar que los procesos de trabajo traen en sí la singularidad de los territorios existenciales, en los cuales se inscriben los trabajadores, pudiendo ser, por ejemplo, territorios existenciales que expresan como valor el acogimiento, el vínculo y el cuidado-cuidador, o lo inverso. $Y$ es esto lo que va a definir si el cuidado que se produce es de un modo o de otro. El hecho de que el territorio existencial habite en el sujeto, significa que donde él esté, sea en la atención básica, en el hospital, en la atención especializada, domiciliaria, etc., va a producir el tipo de cuidado que se inscribe en su universo como una ética -el modo de ser en el mundo- a ser construida. Por lo tanto, lo que define el perfil del cuidado no es el lugar físico donde se realiza el cuidado, sino el territorio existencial en el cual el trabajador se inscribe como sujeto ético-político, y que anda con él, donde él opere su proceso de trabajo.

Ese proceso es intenso, dinámico y tensionado por sucesivos y continuos procesos de cambio. La producción subjetiva de la realidad social se da a través de movimientos de desterritorialización y reterritorialización de los propios sujetos que producen el cotidiano: el funcionamiento social. $Y$ en ese proceso, los sujetos operan cada movimiento con intensidades diferentes, pudiendo desterritorializarse, lo que significa romper con el lugar de origen y por lo tanto adoptar nuevos territorios existenciales, ética y políticamente identificados con la producción de una nueva realidad social; por otro lado, pueden no completar el movimiento de desterritorialización, retornando al lugar de origen y así no producir cambios sociales, eternizando un estatus conservador de funcionamiento social. 
La desterritorialización puede darse por "coeficientes" diferentes, respetando una cierta graduación de sentidos para las rupturas a ser realizadas. Por último, la autora nos revela en el recorrido de su cartografía una dinámica de producción subjetiva de la realidad, que pone de manifiesto los sentidos, la multiplicidad, la complejidad de la acción humana en el ámbito de la micropolítica, su expresión y construcción social.

En líneas generales la cartografía es un método, pero al mismo tiempo es un anti-método porque no tiene pretensión de verdad, no se propone como modelo a ser seguido, y por eso mismo sustenta la idea de que hay un método para cada objeto, o sea: la cartografía es un método "ad hoc". Esto se basa en el supuesto de que es extremadamente complejo para una evaluación de los servicios de salud revelar procesos de producción de subjetividades, haciendo uso de la observación de agenciamientos de deseo, de factores de afectivación, de dispositivos colectivos de producción social, sobre la base de que hay una producción subjetiva de la realidad social. Siendo así, la subjetivación produce nuevos territorios existenciales, y posibilita la invención de nuevos mundos en un proceso simultáneo de invención de sí mismo (5). Entendemos que los instrumentos cartográficos tienen la suficiente sensibilidad como para captar la realidad de la producción del cuidado en su forma más próxima de lo real, cerca del caos, en los encuentros donde se producen los flujos de intensidades, la producción de afectos, las tecnologías que revelan la cara organizada de los saberes aplicados a la producción del cuidado; en síntesis, la cartografía posibilita adentrarse en el complejo mundo singular y al mismo tiempo múltiple del cuidado en salud. Pero por otro lado, la realidad social puede manifestarse, no en la producción, sino en la reproducción, en procesos de captura subjetiva de los sujetos, en los que la ética del cuidado está apresada por la normatividad de la vida y del trabajo, por la repetición de sentidos, la desfiguración de los signos, produciéndose así un borramiento del campo de visión del "ojo vibrante". Esto es lo que la cartografía debe analizar, tanto en el plano de intensidades de producción de la vida, como en el plano de captura molar de la existencia.

\section{CARTOGRAFIAR LA PRODUCCIÓN DEL CUIDADO EN LA LÍNEA DEL "TRABAJO VIVO EN ACTO"}

La construcción del Sistema Único de Salud (SUS) fue un acontecimiento que produjo un gran impacto en el concepto de salud, del derecho a la asistencia, al imprimir en la vida cotidiana de los servicios de salud la idea de ciudadanía. Puso en escena nuevos modos de trabajar en salud y, sobre todo, la comprensión de que el escenario de prácticas de los servicios es multiprofesional, y que el cuidado se construye siempre en relación con el otro, sea un trabajador o un usuario.

EI SUS fue por lo tanto un acontecimiento de gran impacto, capaz de disparar procesos de subjetivación, es decir, producción colectiva de nuevas subjetividades. La subjetividad es social e históricamente construida, y agenciada en base a acontecimientos, encuentros, las múltiples vivencias que un sujeto tiene en su experimentación e interacción social. Lo que queremos decir es que el encuentro de un trabajador individual o colectivo con un acontecimiento -como lo fue la construcción del SUS- puede disparar en él la producción de nueva subjetividad, o sea, una nueva forma de significar el cuidado e interactuar con su construcción social, un "factor de afectivación" (b), algo que afecta a los que están presentes en el escenario de impacto del SUS, y de ese modo produce nuevas subjetividades basadas en ese encuentro.

EI SUS se desarrolló como producción teórica, práctica y subjetiva, a partir del campo de la vigilancia a la salud. Este referencial, instrumentalizado por la epidemiología, produjo un gran campo ordenador de experiencias y modelado de los servicios de salud, que se hicieron notorios en Brasil, como el de Sistemas Locales de Salud (9), Distritos Sanitarios (10); Ciudades Saludables (11), todos vinculados al campo de la vigilancia de la salud y con un fuerte componente de la Promoción de la Salud (12). Todos los escenarios de producción del por entonces recién nacido sistema de salud de Brasil, el SUS, la formación, las redes de servicios, la investigación y publicación en el área, en fin, todos los segmentos fueron agenciados por aquel territorio 
de saberes y prácticas, sobre el cual se edificaron las bases para el sistema de salud.

Por otro lado, en la multiplicidad de campos que constituyen la salud, se constituyó otro territorio de referencia que influye sobre la producción subjetiva en el área: el modelo "anátomo-clínico" de estructuración de los saberes y prácticas de salud, que toma como base el Informe Flexner (c), principal dispositivo para la reorganización de los referenciales de formación médica y de las diversas profesiones de salud en el siglo XX. Dado el avance tecnológico y la tensión creada por el complejo médico-industrial de la organización de los servicios basada en el alto consumo de insumos, el modelo asistencial que nace de esta corriente, fue asumiendo la característica hegemónica de un cuidado más volcado a la "producción de procedimientos" (15).

La vigilancia y la clínica como campos de saberes y prácticas convivieron lado a lado en la construcción del SUS, pero no de forma solidaria, sino como campos opuestos. Esta oposición no es natural, fue construida imaginariamente por los que idearon la reforma sanitaria; es decir, los sujetos que formularon el campo de la salud, produjeron una división simbólica y discursiva entre el modelo "preventivista" originario del campo de la vigilancia de la salud, y el modelo "clínico" centrado en las prácticas "curativas". Obviamente que este caso específico, se trata de una representación asociada a la clínica flexneriana. Pero insistimos en señalar que no necesariamente debería significar eso, pues la clínica puede tener diversas connotaciones y prácticas, $y$ puede incluso constituir diferentes campos. Sin embargo, esta dicotomía se produjo principalmente porque la clínica, en el caso de la reforma sanitaria, fue asociada al modelo biomédico.

Esa falsa polaridad entre vigilancia y clínica es transmitida por los diversos dispositivos de construcción del SUS: la formación, educación en salud, regulación del sistema, protocolos de servicios, etc.; y de ese modo se forma en el ámbito de la producción del cuidado una subjetividad que opera colectivamente en la producción de servicios altamente involucrados con la promoción y prevención de la salud, y por otro lado poco comprometidos con una práctica clínica cuidadora, que cuando se produce, opera centrada en la lógica burocrática de la programación en salud.
En la conducción de los servicios de salud se percibe una lógica determinada por la razón, los saberes constituidos, y por otro lado, otra lógica que opera a través de las subjetividades formadas en el contexto de la construcción del SUS. Si aquella es fácilmente organizable a través de los protocolos y la educación en salud es posible producir una estandarización de los procedimientos. Sin embargo, el cuidado en acto es proporcionado a través de los afectos (d), que circundan los encuentros entre trabajadores y usuarios. Este escenario está determinado sobre todo por las singularidades, por lo tanto, los modelos que allí operan pueden ser tantos, como sujetos haya en ese encuentro. Es en este escenario marcado por el caos, en el que de hecho se produce el cuidado, y se abre al agenciamiento de nuevas prácticas y a la creatividad de los propios trabajadores.

El desarrollo del SUS produjo ciertas paradojas que sirven como analizadores del propio modelo tecnoasistencial que se creó. Un problema-analizador se vincula a la universalidad de acceso, que si bien se inscribió como el principal principio del SUS, los servicios aun conviven con grandes esperas, filas, y en la mayoría de los servicios dispositivos racionadores como la selección de usuarios para el ingreso a los servicios, turnos para procedimientos, etc. Se observan incluso procesos de trabajo fragmentados, aunque exista un discurso de trabajo en equipo; saberes técnicos supuestamente omnipotentes pero poco eficaces para satisfacer las necesidades de los usuarios; relaciones jerárquicas en el equipo de salud; en síntesis, las contradicciones revelan fuertes tensiones en las redes de atención de la salud.

El proceso de buscar la superación de este escenario, en el ámbito del caso brasileño, se fue produciendo a lo largo del tiempo. Inicialmente con el cuestionamiento por parte de diversos autores de la salud colectiva acerca de cómo se constituían los territorios de captura en el campo. A modo de ejemplo, se puede mencionar la medicalización de la sociedad, es decir, la constitución, en el ámbito social, de un pensamiento centrado en el modelo biológico y de una construcción de los servicios médicos a gran escala, con el objetivo de la expansión del mercado de trabajo médico, sobre todo luego del advenimiento de la medicina comunitaria en 
EE.UU., en los años '50 y '60 (16). Es importante mencionar también la construcción de esta hegemonía en el ámbito de las instituciones brasileñas, descrita por Luz (17 p.50-51) como la producción de una racionalidad médica hegemónica en el Estado y en la sociedad.

El desarrollo del SUS se compone de múltiples formulaciones, y el tema de la clínica pasa a ser valorizado en el ámbito de la reforma sanitaria, junto con la idea general de invertir en la micropolítica de los procesos de trabajo para la consolidación de procesos de cambio en la salud. Estas formulaciones, producidas principalmente en la década del '90 por Gonçalves (18), Cecílio (19), Campos (20) y Merhy (6), abrieron un nuevo campo de investigación y de intervención sobre los escenarios de producción del SUS como proyecto técnico-político y construcción subjetiva de una determinada ética del cuidado.

EI SUS se va formando en base a una multiplicidad de saberes, acciones, técnicas y políticas, conformando un escenario diverso y al mismo tiempo rico en referenciales que se constituyen en campos de apuesta para la formación de determinado modelo tecnoasistencial. Estos esfuerzos son, sobre todo, la expresión de los deseos de colectivos de trabajadores, formuladores de políticas y usuarios, formados en la estela de la reforma sanitaria y en los acalorados debates de los cambios estructurales vividos en Brasil y en particular en la salud en los años '80 y '90.

Vimos aquí que el deseo es el núcleo propulsor de la producción social de los sujetos individuales y colectivos, y crea las subjetividades que expresan singularidades, o sea, el modo singular de percibir y actuar en el mundo en un determinado tiempo y espacio. Por lo tanto, esto puede modificarse todo el tiempo, y un mismo sujeto puede expresar diversas singularidades, dependiendo del tiempo-espacio en el que esté inserto y de los factores de afectivación a los que se exponga. La expresión del medio social se percibe como absolutamente compleja, dinámica e identificada con multiplicidades. EI SUS, por lo tanto, es expresión de las diversas formaciones que le dieron sentido: territorios marcados por la tradición de la prevención y promoción de la salud, por la clínica centrada en la investigación biológica, por los procesos de trabajo centrados en el acto prescriptivo y poco relacionales, por los cuidados acogedores, por los vínculos que se forman entre trabajadores y de ellos con los usuarios; en fin, hay una infinidad de agenciamientos que conforman el escenario complejo de producción, pero al mismo tiempo son la manifestación del mundo real, su expresión más nítida, bajo la lente que las interpretaciones de la realidad tienen el hábito de colocar y, muchas veces, enmascaran la propia realidad o modifican su imagen.

Según Rolnik (3) el territorio existencial es un referencial que forma sentidos e identidades en el sujeto, es decir, singularidades que operan en el mundo de la vida en general. En el caso de la salud podemos decir que la producción del cuidado se da siempre en base a un trabajador individual o colectivo que opera su proceso de trabajo teniendo como referencia sus territorios existenciales. El movimiento de cambio en la salud presupone procesos de desterritorialización -o sea, la ruptura con el viejo territorio- y movimentos de reterritorialización, buscando nuevas identidades existenciales que van a demandar nuevas prácticas de cuidado. La desterritorialización presupone agenciamientos, es decir, procesos de cambio que son conflictivos, dolorosos, permeados por idas y vueltas en las que el sujeto está todo el tiempo enfrentándose a sí mismo y al nuevo territorio que se anuncia; es algo parecido a "perder el suelo", a una muerte de sí, hacia la búsqueda de otro suelo y de sí mismo bajo nuevos referenciales de vida y producción.

En el campo de la salud, de forma hegemónica, se estructuran escenarios de un devenir capitalístico (e) de la producción del cuidado, marcado por un modelo tecnoasistencial centrado en procedimientos y un alto consumo de insumos. La construcción de un devenir solidario para el SUS presupone la existencia de dispositivos capaces de agenciar la construcción de nuevos saberes y prácticas que resignifiquen el trabajo en salud y, sobre todo, el cuidado. El agenciamiento de deseos capaces de operar en la construcción de un nuevo SUS presupone el enfrentamiento con los territorios actualmente estructurantes de los servicios de salud y, sobre todo, una ruptura con su devenir capitalista y capitalístico.

Constantemente hay diversos territorios constituyéndose en el escenario del Sistema Único de Salud. Los agenciamientos subjetivos, en su movimiento de producción del mundo, 
promueven la deconstrucción y al mismo tiempo nuevas formaciones de territorios en el ámbito de la micropolítica del proceso de trabajo. Esto solo es posible porque el trabajo en salud es dependiente del trabajo vivo en acto $(6,21)$ lo que posibilita, dada la libertad de acción en salud, muchos movimientos de idas y vueltas, territorializaciones y desterritorializaciones, composición y decomposición de mundos. Este es un movimiento continuo de descubrimientos que se originan en el movimiento cotidiano de producción del SUS. Es importante enfatizar que en la misma medida en que el trabajador individual y colectivo produce el mundo del cuidado en salud, es producido a su vez por este mismo mundo, por los afectos circulantes en las relaciones que se entablan entre sí y los otros. El trabajador de la salud es al mismo tiempo productor y producto de determinados modelos tecnoasistenciales. "Cada uno de nosotros pasamos por las más variadas micropolíticas y, en cada una de ellas, cambia nuestra manera de pensar, sentir, percibir, actuar: cambia todo" (3 p.55).

Cambiar el modo de producción del cuidado presupone, desde nuestro punto de vista, además de un cambio en el proceso de trabajo, un proceso de desterritorialización de los trabajadores y usuarios del SUS, teniendo como referencia que los trabajadores operan a partir de territorios existenciales. Ese territorio existencial no es físico, sino que se encuentra dentro de cada trabajador, organizado por su subjetividad. Por lo tanto, un cambio de carácter estructural y duradero de los procesos de trabajo requiere la producción de una nueva subjetividad del trabajador. Este proceso es difícil, complejo y doloroso, pues significa romper con los modos estructurados de trabajo y producción; y en este caso, el trabajador se va a encontrar con el mundo tal como es: caótico pero potente en las singularidades que se forman y encuentran sinergia para el cuidado en salud. El cambio en la producción del cuidado está marcado por nuevas subjetividades activas en la producción del cuidado, de un modo de trabajar en salud que tiene como centro el campo relacional, abriendo el encuentro con los usuarios a espacios de habla, escucha, miradas y signos que tienen sentido para ambos, trabajador y usuario, y forman el centro del proceso de trabajo. Hasta el momento, los procesos de cambio han sido siempre parciales y no consiguen producir una desterritorialización del modelo médico hegemónico, que opera el cuidado centrado en la lógica de la producción de procedimientos. La reestructuración productiva,

\footnotetext{
...es la resultante del cambio en el modo de producir el cuidado, generada a partir de innovaciones en los sistemas productivos de la salud, que impactan en el modo de fabricar sus productos, y en su forma de asistir y cuidar a las personas y a los colectivos poblacionales. (23)
}

Aunque represente un momento de cambio en el modo de producir el cuidado, la reestructuración se da en los límites del actual modelo; es decir, los agenciamientos capitalísticos que operan en la producción de la salud continúan activos en las subjetividades actuales, incluso con un cambio en el proceso de trabajo.

Para una "transición tecnológica" sería necesario que la reestructuración productiva avance en el sentido de romper con las actuales estructuras del modelo biomédico, del proceso productivo centrado en el acto prescriptivo, organizando procesos de trabajo más relacionales. Si un proceso de reestructuración no llega a este nivel deja de ser instituyente para instituirse en un territorio fijo, que realiza una producción diferente desde el punto de vista del modo actual de producir el cuidado, pero que no rompe con sus fundamentos, como por ejemplo con el alto consumo de tecnologías duras, el plano secundario de la dimensión relacional del cuidado en salud y las intersubjetividades que operan en la producción subjetiva del cuidado. Ese proceso, recrudece, inmoviliza y endurece las estructuras que componen el proceso de trabajo, impidiendo agenciamientos del deseo que se manifiestan y están activos en la construcción de nuevos territorios de las prácticas en salud. 


\section{AGRADECIMIENTOS}

Este artículo forma parte de las conclusiones de la investigación "A produção do cuidado na rede básica de saúde" desarrollada entre los años 2004 y 2008 en el estado de Bahía, Brasil, con el apoyo de la Fundação de Amparo à Pesquisa da Bahia (FAPESB) y el Conselho Nacional de Desenvolvimento Científico e Tecnológico (CNPq).

\section{NOTAS FINALES}

a. Ciertas citas reproducidas aquí en español corresponden a traducciones libres de textos publicados en portugués.

b. Sobre el "factor de afectivación", ver Rolnik (3).

c. El denominado modelo flexneriano hace referencia al modelo de enseñanza médico implementado a partir del Informe Flexner (13), que sugería una formación que tuviera como eje "la necesidad de enlazar la enseñanza con la investigación en las ciencias biomédicas", y que derivó en un modelo de práctica médica centrada en el cuerpo anátomo-fisiológico, teniendo como principal referencia al hospital (14 p.92-93).

d. "Afectos" tiene aquí el sentido dado por Espinosa, y se refiere a la capacidad de afectar y ser afectado a partir del encuentro. La afectación que aporta positividades ("alegría"), produce mayor potencia de acción en el mundo y el que aporta negatividades ("tristeza"), produce menor potencia. Citado en Deleuze (8).

e. A diferencia de capitalista, que refiere a un sistema económico, capitalístico significa un modo de vida, trabajo, existencia, subjetivamente centrado en referencias subjetivamente orientadas por la organización social del consumo. En la producción del cuidado en el campo de la salud se podría decir que lo capitalístico se relaciona con un proceso de trabajo altamente consumidor de procedimientos, en detrimento de procesos más relacionales, es decir centrados en territorios existenciales que traen lógicas propias de la subjetividad capitalística. Ver Guattari y Rolnik (22).

\section{REFERENCIAS BIBLIOGRÁFICAS}

1. Deleuze G, Guattari F. Mil mesetas: capitalismo y esquizofrenia. Valencia: Pre-Textos; 1988.

2. Deleuze G, Guattari F. El Anti Edipo. Capitalismo y esquizofrenia. Buenos Aires: Paidós; 2007.

3. Rolnik S. Cartografia sentimental. Porto Alegre: Editora da UFRGS; 2006.

4. Osório C, Machado JMH, Minayo Gomez C. Proposição de um método de análise coletiva dos acidentes de trabalho no hospital. Cadernos de Saúde Pública. 2005;21(2):517-524.

5. Kastrup V. O funcionamento da atenção no trabalho do cartógrafo. Psicologia \& Sociedade. 2007;19(1):15-22.

6. Merhy EE. Em busca do tempo perdido: a micropolítica do trabalho vivo em saúde. En:
Merhy EE, Onocko R, organizadores. Agir em saúde: um desafio para o público. San Pablo: Hucitec; 1997.

7. Merhy EE. Salud: cartografía del trabajo vivo. Buenos Aires: Lugar Editorial; 2006.

8. Deleuze G. Espinosa: filosofia prática. San Pablo: Editora Escuta; 2002.

9. Paim JS. Saúde da Família: espaço de reflexão e contra-hegemonia. Interface - Comunicação, Saúde, Educação [Internet]. 2001;5(9):143-146 [citado 10 mar 2010]. Disponible en: http://www.interface.org.br/revista9/debates2.pdf

10. Mendes EV, organizador. Distritos Sanitários. San Pablo: Hucitec; 1994.

11. Akerman $M$, Mendes $R$, organizadores. Avaliação participativa de municípios, comunidades e ambientes saudáveis: a trajetória brasileira - memória, reflexões e experiências. San Pablo: Midia Alternativa; 2006. 
12. Buss PM. Promoção da saúde e qualidade de vida. Ciência \& Saúde Coletiva. 2000;5(1):163177.

13. Flexner A. Medical education in the United States and Canada: a report to the Carnegie Foundation for the Advancement of Teaching. Nueva York: Carnegie Foundation for the Advancement of Teaching; 1910.

14. Nogueira RP. Perspectivas da qualidade em saúde. Río de Janeiro: Qualitymark; 1994.

15. Merhy EE. A perda da dimensão cuidadora na produção da saúde. Uma discussão do modelo assistencial e da intervenção no seu modo de trabalhar a assistência. En: Campos CR, Malta DC, Reis AT, Santos AF, Merhy EE, organizadores. Sistema Único de Saúde em Belo Horizonte: reescrevendo o público. Belo Horizonte: Xamã; 1998.

16. Donnangelo MC. Saúde e sociedade. San Pablo: Duas Cidades; 1976.

17. Luz MT. As Instituições Médicas no Brasil: instituição e estratégia de hegemonia. 2da ed. Río de Janeiro: Graal; 1981.
18. Gonçalves RBM. Tecnologia e organização social das práticas de saúde. San Pablo: Hucitec; 1994.

19. Cecílio LCO, organizador. Inventando a mudança na saúde. San Pablo: Hucitec; 1994.

20. Campos GWS. Reforma da reforma, repensando a saúde. San Pablo: Hucitec; 1992.

21. Franco TB, Merhy EE. Mapas analíticos: una mirada sobre la organización y sus procesos de trabajo. Salud Colectiva. 2009;5(2):181-194.

22. Guattari F, Rolnik S. Micropolítica: cartografias do desejo. Petrópolis: Editora Vozes; 1999.

23. Merhy EE, Franco TB. Reestruturação produtiva em saúde. En: Dicionário da educação profissional em saúde. Río de Janeiro: Escola Politécnica Joaquim Venâncio, Fiocruz; 2006. p. 225-230.

\section{FORMA DE CITAR}

Franco TB, Merhy EE. El reconocimiento de la producción subjetiva del cuidado. Salud Colectiva. 2011;7(1):9-20.

Recibido el 12 de septiembre de 2010

Aprobado el 18 de noviembre de 2010 